\title{
The trabecular wall of Schlemm's canal: a study of the effects of pilocarpine by scanning electron microscopy
}

\author{
I. GRIERSON, W. R. LEE, H. MOSELEY, ${ }^{1}$ AND S. ABRAHAM \\ From the University Departments of Ophthalmology and Pathology, The University, Glasgow, and \\ ${ }^{1}$ West of Scotland Health Boards Department of Clinical Physics and Bio-Engineering, Glasgow
}

SUMMARY The scanning electron microscope was used to study the endothelium lining the trabecular wall of Schlemm's canal in 10 human eyes enucleated in treatment of choroidal melanomas. The eyes of 5 patients had been treated before enucleation with pilocarpine drops, and the remaining 5 were untreated and served as controls. In the pilocarpine-treated tissue there was an increased prominence of cellular bulges in the endothelial monolayer, and the endothelial pores were both larger and more frequent than in the untreated eyes. By the use of Poiseuille's formula it was calculated that the resistance offered by the pore system to the drainage of aqueous in the pilocarpine-treated group was approximately one-ninth of that in the control series.

In a previous investigation the present authors showed by light microscopy and transmission electron microscopy (TEM) that the incidence of giant vacuoles and transcellular pathways in the endothelium of Schlemm's canal was greater in human eyes which had been treated with topical pilocarpine than in a suitable control group of a similar age range (Grierson et al., 1978). This finding was in disagreement with the results of a previous quantitative TEM investigation (Holmberg and Bárány, 1966) in which it was shown that vacuole counts in the endothelium of Schlemm's canal were lower after the topical application of pilocarpine. The experiment of Holmberg and Bárány was conducted on a group of cynomolgus monkeys, and both gross facility and net outflow were increased by the action of the drug. In an attempt to explain their findings Holmberg and Bárány proposed that the vacuolar flow pathways had become less tortuous and that straightening out of the vacuolar channels would account both for the decrease in incidence in single sections and for the increased rate of drainage.

Svedbergh (1976), however, has criticised the use of TEM in the assessment of changes in flow pathways through the lining endothelium of Schlemm's canal. This criticism is largely based on the fact that only a small proportion of giant vacuoles are transcellular channels (Kayes, 1967; Tripathi, 1968, 1971, 1974; Inomata et al., 1972; Grierson and Lee,

Address for reprints: Dr W. R. Lee, Tennent Institute of Ophthalmology, University of Glasgow, 38 Church Street, Glasgow G11 6NT
1975; Grierson et al., 1978), and it is possible to find transcellular channels which are not associated with a vacuolar swelling (Inomata et al., 1972; Grierson and Lee, 1975).

Quantitative data on the luminal openings of vacuolar and nonvacuolar transcellular channels can be obtained by examining the endothelium lining the trabecular aspect of Schlemm's canal with the scanning electron microscope (SEM). This technique provides a useful means of examining relatively large areas of exposed canal endothelium and overcomes the sampling problem associated with TEM. It was therefore considered appropriate to apply scanning electron microscopy to the study of the material which was used in the initial light microscopy and TEM investigation of the effects of pilocarpine in the human outflow system. In particular, the analysis was directed towards the variation in incidence and size of the luminal pores in the endothelium of the treated and untreated tissue.

\section{Materials and methods}

The tissue for this investigation came from 10 patients with an ostensibly normal anterior segment, and each eye was enucleated in treatment of a choroidal melanoma. With 5 patients the eye to be enucleated was subjected to a topical application of 2 to $4 \%$ pilocarpine on 4 separate occasions at 6-hourly intervals before surgery. The remaining 5 patients were untreated, and the tissue served as a control. On enucleation the eyes were immersed in 2 to $4 \%$ glutaraldehyde in phosphate buffer. There- 
after blocks of limbal tissue were removed, washed in buffer, postfixed in $1 \%$ buffered osmium tetroxide, and washed again in phosphate buffer.

The technique for dissection of the trabecular wall of Schlemm's canal has been described previously (Lee, 1971). With a ground razor blade a cut was made from the anterior limit of the canal across the peripheral cornea and into the supraciliary space from the posterior portion. The dissection leaves 2 pieces of tissue, one which shows the surface of the scleral sulcus and the other the endothelium of the trabecular wall.

The tissue was freeze-dried in a Balzars Micro BA3 unit which maintained a vacuum of $10^{-5}$ Torr. Before water extraction the tissue was passed through liquid Arcton $^{12}$ and frozen in liquid nitrogen. The dried tissue was coated with gold in a Polaron Sputter Coater E5000 and examined in a Cambridge Stereoscan S600.

For the quantitative analysis of endothelial porosity coded pieces of tissue from pilocarpine treated and untreated eyes were processed in parallel to minimise variation in preparation. Mapping photographs of the endothelial monolayer were taken at a screen magnification of $\times 3000$ and enlarged to a print size which corresponded to an area of $2500 \mu \mathrm{m}^{2}$. The number of cellular bulges and surface openings were recorded on coded photomicrographs, and the total area examined in each eye was between 250000 and $400000 \mu \mathrm{m}^{2}$. Maximum pore widths were measured from sets of prints which corresponded to a surface area of $100000 \mu \mathrm{m}^{2}$ with the use of a $\times 7$ magnifier which incorporated a calibrated graticule.

\section{Results}

Examination of the dissected portions of Schlemm's canal from both the treated and untreated eyes showed that the general topography of the endothelium lining the trabecular aspect of the canal was similar to previously published findings (Hoffmann and Dumitrescue, 1971; Bill and Svedbergh, 1972; Segawa, 1973). In some regions the endothelium was flat, whereas in others there were distinct ridges, humps, and areas of disruption where septa had been torn. The individual cells of the endothelial monolayer were elongated and spindle shaped, and each had a prominent central ovoid bulge (Fig. 1).

Each bulge contained the endothelial cell nucleus and possibly a giant vacuole, but, in adequately prepared tissue, identification of the giant vacuoles was speculative except where the delicate vacuolar shell was mechanically disrupted during dissection to reveal its hollow interior. If this disruption was

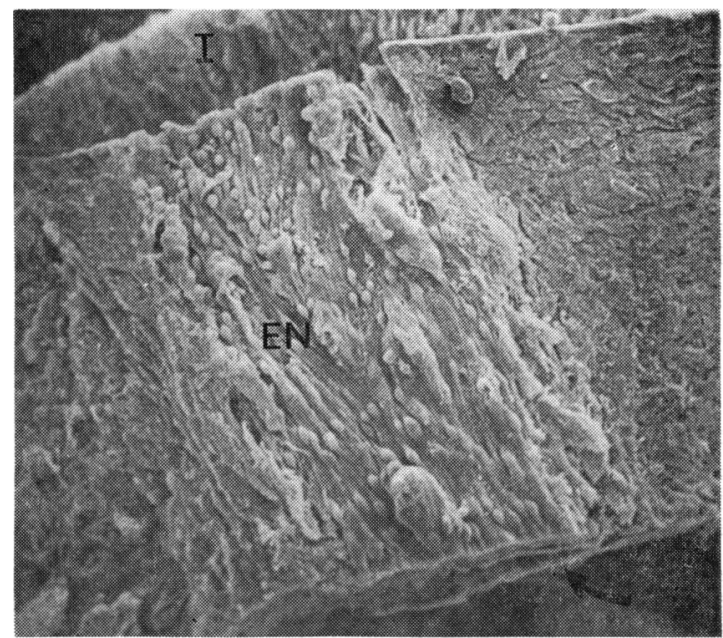

Fig. 1 A dissection of the endothelium (EN) lining the trabecular aspect of Schlemm's canal in an untreated eye. The trabecular meshwork is indicated by an arrow. The iris (I) is seen beneath the trabecular meshwork (arrow) $(\times 120)$

sufficiently extensive the meshwork pore at the base of the cystic cavity was exposed (Fig. 2).

Openings with a smooth outline were found both on and off the bulges. As in previous reports of Bill (1970), Bill and Svedbergh (1972), Segawa (1973), and Lee and Grierson (1975), the former were considered to be the lumen pores of giant vacuoles and the latter to be the openings of nonvacuolar transcellular channels (Fig. 2). The distributions of both types of pore were not uniform, and areas of particular abundance and also areas of deficiency were common to both the eyes treated with pilocarpine and the untreated tissue. Surface pitting was also found to a greater or lesser extent, both on and off the bulges. This was an artifact which may have been produced by the crystallisation of surface deposits (plasma) during the freeze-drying, and this was a troublesome feature because the largest pits could have been mistaken for pores. Therefore the identification of true pores was not always clear-cut, but with care and critical evaluation most of the artifacts could be eliminated. In addition the study was essentially relative, and it was assumed that the preparation hazards were equally applicable to the treated and the untreated tissues.

There were qualitative differences in the endothelial bulges which protruded from the endothelial cells of the monolayer between the treated and untreated tissue. In the pilocarpine treated tissue the cellular bulges occupied a larger portion of the monolayer. They protruded further into the canal and were often seen as smooth-walled spherical 


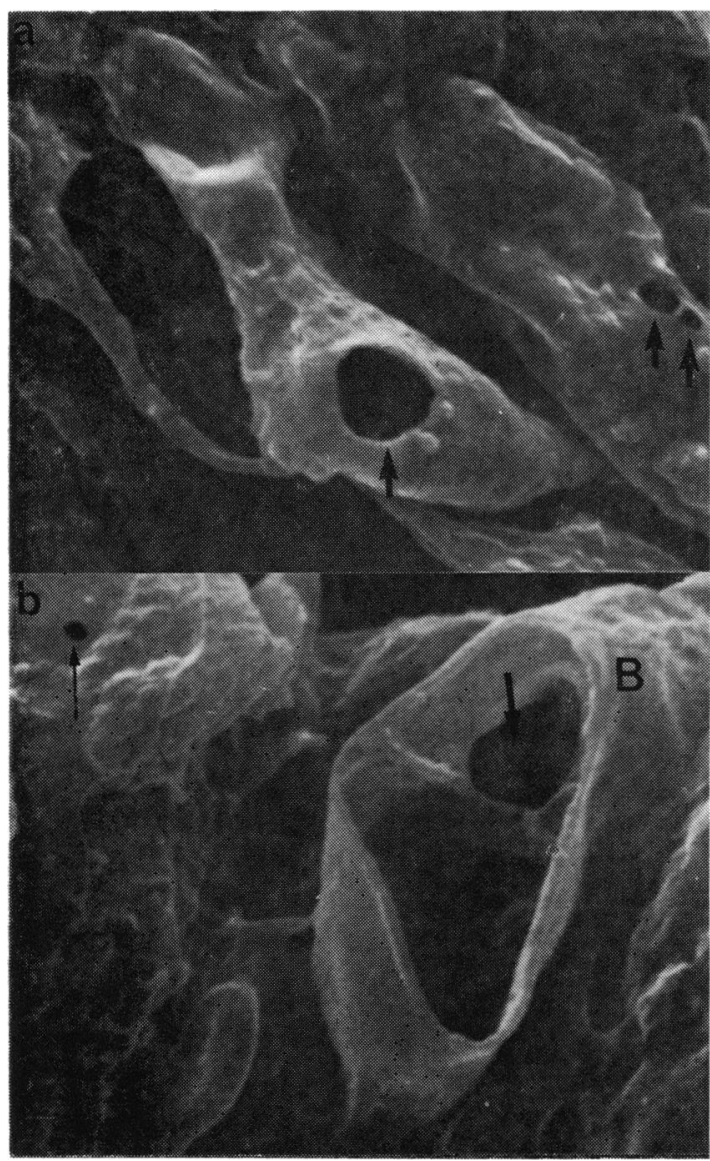

Fig. 2 (a) Pores in endothelial bulges (arrows) from the trabecular wall of Schlemm's canal in an untreated eye ( $\times 3800)$. (b) A bulge (B), which has a large artifactual opening, in the trabecular wall endothelium of Schlemm's canal in an untreated eye. The bulge appears to be hollow and a meshwork pore (arrow) is evident at the base of the cavity. Note the non-bulge pore elsewhere in the endothelium (small arrow) $(\times 3800)$

grape-like structures, whereas the bulges in the untreated tissue were usually less obtrusive, more ovoid, and sometimes had a rough crenated surface (Fig. 3). The bulges in the treated tissue were more prone to total or partial collapse (the action of surface tension forces during the drying process) and to artifactual tearing than in the untreated tissue. Presumably this was due to the presence of particularly large giant vacuoles with delicate and therefore vulnerable cytoplasmic shells.

It was our impression that pores both on and off the bulges were more in evidence in the pilocarpinetreated series than in the untreated group. However, there was considerable variation within both groups, and a quantitative analysis was undertaken to determine in as precise a manner possible the effects of pilocarpine on the canal pores of the endothelial flow pathways.

\section{QUANTITATIVE ANALYSIS}

Three structural features were counted in the endothelial monolayer: (a) bulges (since some contained giant vacuoles), (b) openings on the bulges, and $(c)$ openings elsewhere on the endothelial cells.

There were three major sources of error or inaccuracy in the quantitative analysis. (a) True pores were defined as openings with a regular and smooth outline to distinguish them from ragged deficits which resulted from the preparation. However, the distinction was not always clear-cut and therefore an overcount was possible. Conversely, true pores may have been damaged and were thus eliminated from the count. (b) Particular difficulty was encountered with pores outside the openings which could have been obscured by grooves and ridges in the monolayer. (c) The analysis was based on viewing from one angle only, so that inevitably a proportion of the openings would have been missed.

In view of these problems in analysis a check was carried out on duplicate photographic prints to assess interobserver differences. The difference was found to be $8 \%$ for counts of bulges and $15 \%$ for counts of endothelial pores. Table 1 shows that there was no significant difference in the numbers of cellular bulges between the treated and untreated groups. However, there were substantially greater numbers of endothelial pores both on and off the bulges to the extent that there were nearly three times as many in the pilocarpine treated as in the untreated group.

Measurements of maximum pore diameters were made without stereoscopic correction. Without correction a pore will appear erroneously smaller than its 'true' size, the error depending on the tilt of the pore relative to the viewing angle and the direction of its long axis relative to the viewing direction. Since this error was common to the measurements of pore dimensions in both groups, comparison between the 2 groups could be justified. There was a much smaller population of pores in the untreated than in the pilocarpine-treated series, and the histograms (Fig. 4) showed that in both groups the populations were not normally distributed but had a distinct skew, with smaller pores predominating. The mean pore diameters in the pilocarpine-treated eyes were slightly larger than in the untreated eyes. The average diameter of the pores in the control group was $0.9 \mu \mathrm{m}$ and in the pilocarpine-treated series was $1 \cdot 3 \mu \mathrm{m}$. 

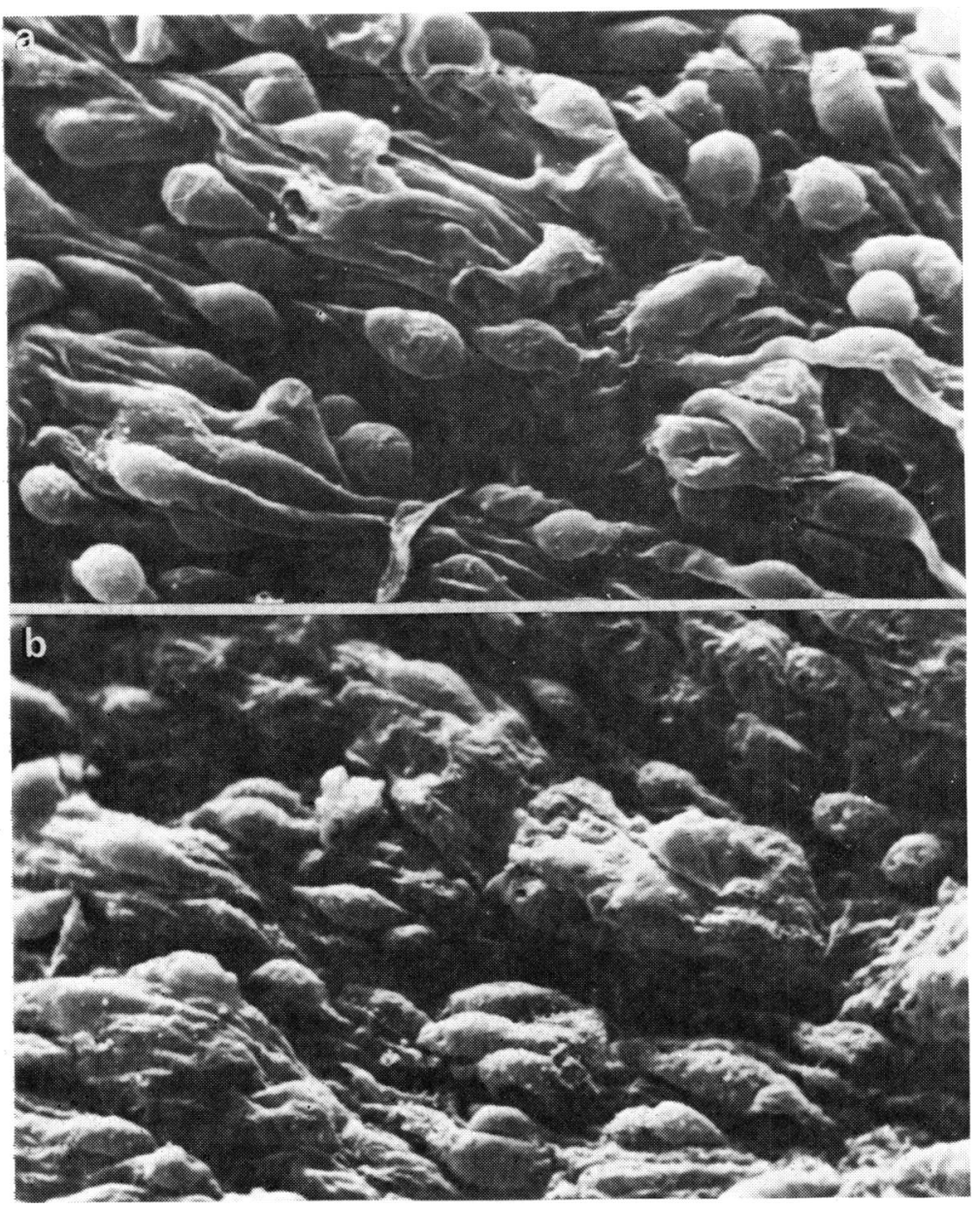

Fig. 3 The endothelium lining the trabecular aspect of Schlemm's canal in (a) a pilocarpine treated and (b) an untreated eye. In the pilocarpine treated tissue the endothelial bulges are smooth walled spherical grape-like structures, whereas the bulges in the control are less obtrusive and more ovoid $(\times 700)$

Table 1 Results (expressed per square millimetre) obtained from the morphological analysis of surface structures in the lining endothelium of the trabecular wall of Schlemm's canal

\begin{tabular}{|c|c|c|c|c|c|c|c|}
\hline $\begin{array}{l}\text { Patient } \\
\text { no. }\end{array}$ & Age & Sex & Bulges & $\begin{array}{l}\text { Pores on } \\
\text { bulges }\end{array}$ & $\begin{array}{l}\text { Percentage bulges } \\
\text { with pores }\end{array}$ & $\begin{array}{l}\text { Pores outside } \\
\text { bulges }\end{array}$ & $\begin{array}{l}\text { Total pores } \\
\text { in endolelium }\end{array}$ \\
\hline \multicolumn{8}{|l|}{ Treated } \\
\hline 1 & 46 & $\mathbf{M}$ & 5690 & 370 & 7 & 130 & 500 \\
\hline 2 & 50 & $\mathbf{F}$ & 4730 & 900 & 19 & 380 & 1280 \\
\hline 3 & 60 & $\mathbf{F}$ & 4630 & 1050 & 23 & 340 & 1390 \\
\hline 4 & 61 & $\mathbf{F}$ & 4670 & 760 & 16 & 210 & 970 \\
\hline 5 & 75 & $\mathbf{M}$ & 4970 & 460 & 9 & 210 & 670 \\
\hline \multicolumn{3}{|c|}{ Mean \pm SD } & $4970 \pm 450$ & $710 \pm 290 *$ & $15 \pm 7$ & $260 \pm 100 \dagger$ & $960 \pm 380^{*}$ \\
\hline \multicolumn{8}{|c|}{ Untreated } \\
\hline 1 & 31 & $\mathbf{F}$ & 5740 & 380 & 7 & 160 & 540 \\
\hline 2 & 63 & $\mathbf{M}$ & 4550 & 200 & 4 & 60 & 260 \\
\hline 3 & 63 & $\mathbf{F}$ & 4130 & 220 & 5 & 90 & 310 \\
\hline 4 & 68 & $\mathbf{F}$ & 3340 & 150 & 5 & 75 & 225 \\
\hline 5 & 75 & $\mathbf{F}$ & 4690 & 280 & 6 & 120 & 400 \\
\hline \multicolumn{3}{|c|}{ Mean \pm SD } & $4490 \pm 880$ & $250 \pm 90$ & $5 \pm 1$ & $100 \pm 40$ & $350 \pm 130$ \\
\hline
\end{tabular}

Levels of significance: $* P<0.001 .+P<0.01$. 
When the pore distributions were pooled and expressed as percentage incidences it was found that there was a distinct difference in the pore distribution profile between the treated and the untreated groups (Fig. 5). Only $3 \%$ of the pore population in the pilocarpine-treated series had a diameter greater than $3.0 \mu \mathrm{m}$, which was the maximum size found in the control group. However, in the untreated group small pores predominated, and approximately $70 \%$ of the population had a diameter of $1 \mu \mathrm{m}$ or less compared with $45 \%$ of the pores in the pilocar-

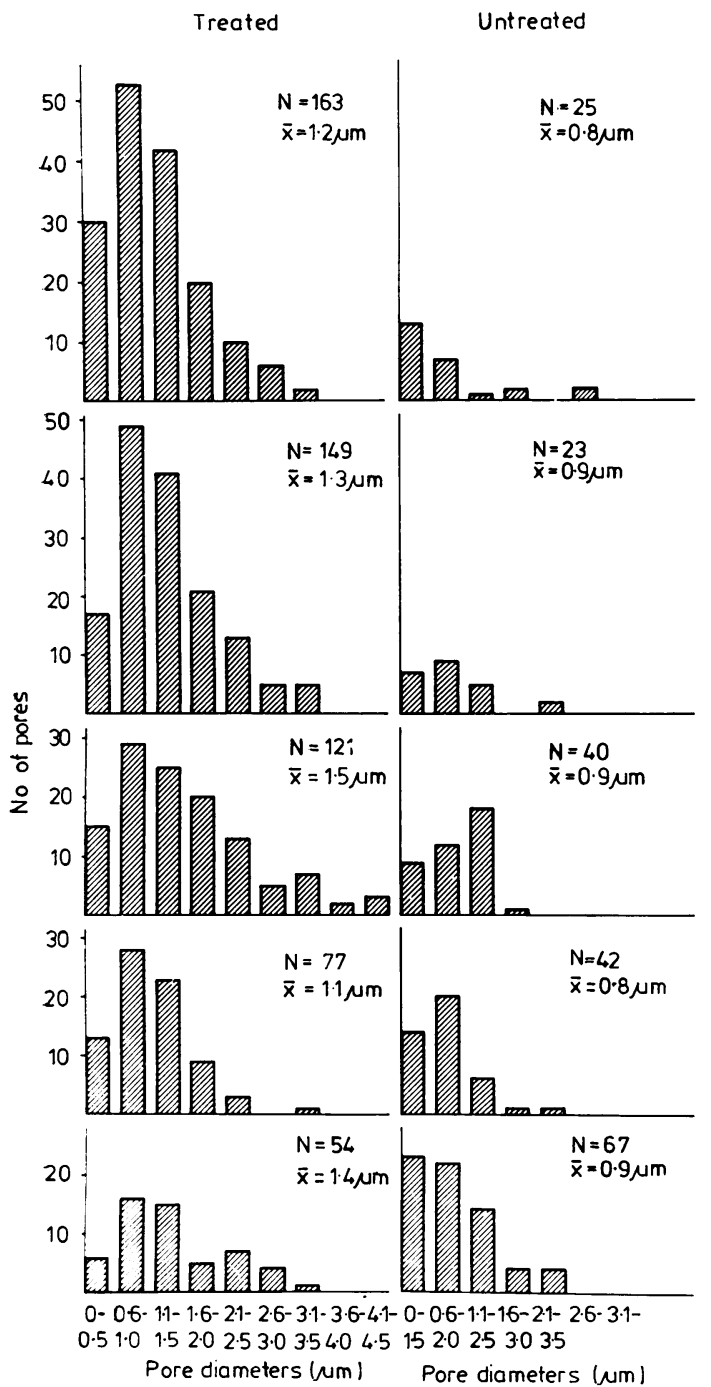

Fig. 4 The distribution of pores on the basis of their diameters, from a sample area of $100000 \mathrm{um}^{2}$ for each of the 10 eyes under investigation. $N$ is the number of pores and $\bar{x}$ is the mean diameter

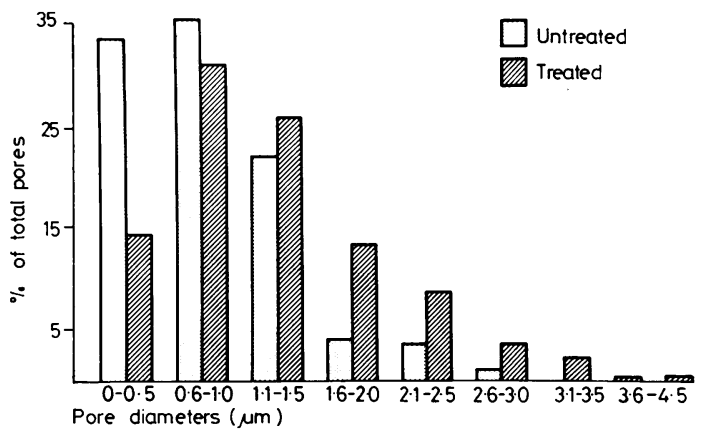

Fig. 5 The percentage incidence of pores in each of the various size categories from the grouped data from the 5 treated and 5 untreated eyes

pine population. On the other hand less than $9 \%$ of the pores from the control population were $1.6 \mu \mathrm{m}$ or greater, which can be compared with a value of $28 \%$ from the pore population in the pilocarpine-treated tissue.

\section{Discussion}

The instantaneous 3-dimensional image of the SEM helped to highlight topographical differences in the endothelial monolayer on the trabecular aspect of Schlemm's canal between the pilocarpine treated and the untreated group. In particular it was shown that the endothelial bulges became more pronounced, and the porosity of the endothelium was increased by the action of the drug. The increased prominence of the cellular bulges was probably the result of the increase in size and incidence of giant endothelial vacuoles in the treated eyes (Grierson et al., 1978). Similar findings are associated with increase in intraocular pressure (Grierson and Lee, 1975).

It was of interest to plot the bulge pore incidence obtained in the present study against the appropriate vacuolar counts published in the previous paper (Grierson et al., 1978). From Fig. 6 it can be seen that high counts of bulge pores are associated with high incidences of giant vacuoles. Although the data are insufficient for meaningful statistical analysis, the trend encourages the assumption that the pores in the bulges are the luminal openings of giant vacuoles.

In both groups it was found that the distribution of endothelial pores was nonuniform, there being areas of relative abundance and other regions where pores could not be found. This observation was considered to be further evidence for the proposal that there are preferential flow pathways through the endothelial meshwork to the overlying endothelial monolayer (Rohen et al., 1967; Bill and 
Svedbergh, 1972; Grierson and Lee, 1974; Grierson, 1976; Lee and Grierson, 1974, 1975; Svedbergh, 1976).

SEM is particularly suited to the study of the relatively rare and sporadically distributed endothelial pores because of the large areas of tissue which can be examined by this facility. Indeed, it was possible to demonstrate quantitatively that the

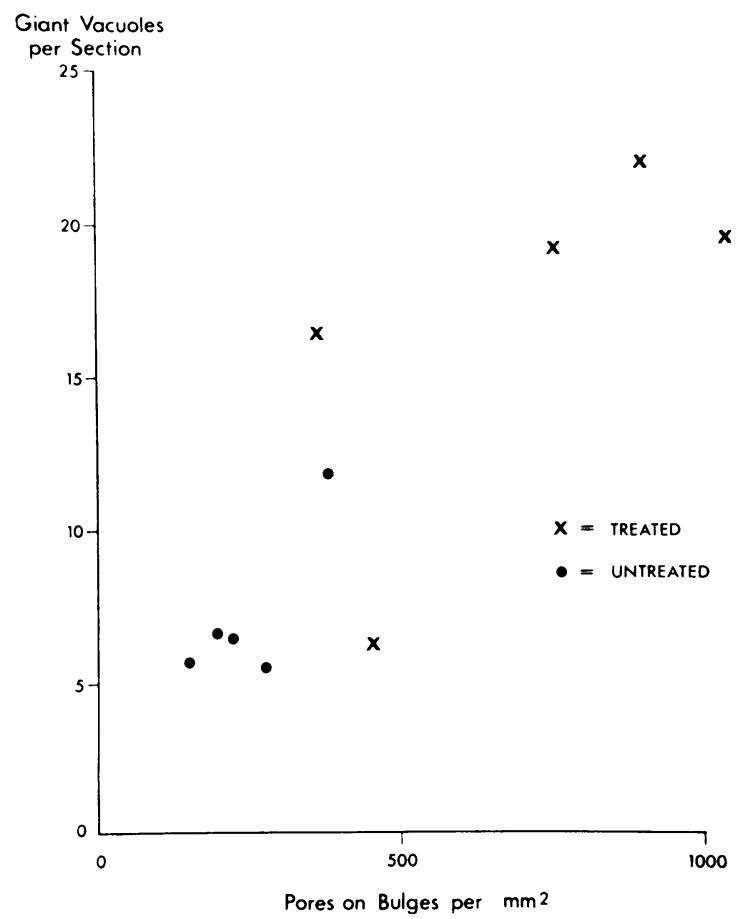

Fig. 6 A scatter diagram which shows the incidence of giant vacuoles per section as derived by light microscopy (Grierson et al., 1978) plotted against the endothelial pore count for the corresponding eye pores were greater in both numbers and size in the pilocarpine-treated series than in the untreated group. Thus the findings of the present investigation do not support the suggestion by Holmberg and Bárány (1966) that the effect of pilocarpine essentially is to change the shape of the flow pathways rather than to produce alterations in their numbers.

However, our results must be interpreted with some caution because pore analysis by SEM is not without hazard. Preparation artifacts, like ice crystal formation, tend to produce holes in the endothelial monolayer and raise the pore count, whereas protein masking, produced by the reflux of blood into segments of Schlemm's canal during the trauma of enucleation, tends to cover pores and depress the pore count. Both factors will influence the absolute values obtained in the analysis, but as they were equally likely to occur in treated and untreated tissue they are not considered to invalidate comparison between the 2 groups. In the light of the problems associated with all aspects of preparation of the tissue for SEM it is of value to make comparison between the data obtained from the human control tissue with that obtained by other authors for normotensive humans and monkeys (Table 2).

The incidence of endothelial bulges found from the present study fits reasonably well with the values previously reported. If it is considered that the total surface area of the trabecular wall of Schlemm's canal is $11 \mathrm{~mm}^{2}$ (McEwen, 1958), then this would give a value from our data of 50000 for the numbers of bulges, and therefore the cells, which make up the monolayer. Bill and Svedbergh (1972) had a much lower estimate of 23000 cells in man, but our figure is very close to the 55000 estimated by Segawa (1973).

The size range of the cellular pores is also in fairly close agreement with the values previously

Table 2 A comparison of data obtained in quantitative SEM studies of the endothelium lining the trabecular wall of Schlemm's canal

\begin{tabular}{|c|c|c|c|c|c|c|}
\hline Author & Species & $I O P$ & $\begin{array}{l}\text { Bulges/ } \\
\mathrm{mm}^{2}\end{array}$ & $\begin{array}{l}\text { Pore } \\
\text { frequency } \\
\text { on bulges }\end{array}$ & $\begin{array}{l}\text { Total pores/ } \\
\mathrm{mm}^{2}\end{array}$ & $\begin{array}{l}\text { Pore size } \\
\text { in } \mu \mathrm{m}\end{array}$ \\
\hline Bill (1970) & Vervet and Rhesus monkey & Normal & 4000 & $29 \%$ & 1200 & $0 \cdot 3-2 \cdot 0$ \\
\hline Lee (1971) & Rhesus monkey & $18-20 \mathrm{mmHg}$ & 6000 & - & - & $0 \cdot 2-1 \cdot 0$ \\
\hline Hoffman and Dumitrescu (1971) & Human & Normal & - & - & - & up to $2 \cdot 0$ \\
\hline Bill and Svedbergh (1972) & Human & Normal & 2000 & - & 1800 & $0 \cdot 15-5 \cdot 0$ \\
\hline Segawa (1973) & Human & Normal & 5000 & $20 \%$ & 1000 & $0 \cdot 15-1 \cdot 5$ \\
\hline $\begin{array}{l}\text { Lee and Grierson (1975), } \\
\text { Grierson (1976) }\end{array}$ & Rhesus monkey & $15 \mathrm{mmHg}$ & 3800 & $15 \%$ & 950 & $0 \cdot 1-4 \cdot 0$ \\
\hline Svedbergh (1976) & Cynomologus monkey & $12 \mathrm{mmHg}$ & - & - & 1640 & $0 \cdot 25-5 \cdot 0$ \\
\hline Present study & Human & Normal & 4500 & $5 \%$ & 350 & $0 \cdot 2-3 \cdot 0$ \\
\hline
\end{tabular}


reported (Table 2), but our values for the total pore incidence and the frequency of pores on bulges are extremely low. To some extent the difference may be accounted for by the quantitative technique adopted in the present study. The pore incidences were determined from micrographs similar to the method adopted by Lee and Grierson (1975) and Grierson (1976) for the study of pressure effects on pore incidence in the rhesus monkey. Bill and Svedbergh (1972) and Svedbergh (1976), who are the only other investigators to use a detailed quantitative approach to the investigation of endothelial pores, obtained particularly high values for pore incidence, and their counts were made directly from the display screen on the SEM. The use of photomicrographs rather than the display screen has several advantages because coding and 'blind' assessment, repeatability testing, and determination of interobserver error are much more easily achieved. However, an undercount is inevitable because 'true' pores are often more difficult to identify in electron micrographs and viewing was from one angle only, so that pores hidden behind the bulges are missed. On the other hand overcounting due to counting from the same area twice would be less likely from photomicrographs than from the display screen.

With the possibility of overcounting in the technique adopted by Bill and Svedbergh (1972) and undercounting by the present approach it would seem reasonable that a value somewhere between 350 and 1800 pores per $\mathrm{mm}^{2}$ (4000 and 20000 respectively for the whole canal) will approach the true incidence in the older human eye. To some extent the large discrepancy is probably also due to the fact that the tissue in both investigations was fixed by immersion without pressure maintenance. It has been reported that immersion fixation is the least satisfactory technique for the study of temporary pressure-sensitive pathways, which may be radically altered in the time interval between enucleation and penetration of the appropriate layers of tissue by fixative (Grierson, 1976; Svedbergh, 1976).

It is of interest to consider the present results in terms of fluid conductance in order to assess the capacity of the canal endothelium to facilitate the drainage of aqueous humour. The fluid conductance, $C\left(\mu \mathrm{l} \mathrm{min}{ }^{-1} \mathrm{mmHg}^{-1}\right)$ of a pore is the flow, $Q$ $\left(\mu 1 \mathrm{~min}^{-1}\right)$ of fluid through the pore per unit drop in pressure, $\Delta P(\mathrm{mmHg})$ across the pore.

$$
C=\frac{Q}{\Delta P}
$$

The mathematical approaches to problems of conductance in such a complex biological system as in the canal endothelium are to be considered in more detail in a later paper. However, the model proposed by Bill and Svedbergh (1972) is suitable for a general estimation of the properties of conductance in the lining endothelium. Bill and Svedbergh represented the pore as an opening $0.3 \mu \mathrm{m}$ long and of various diameters as measured.

Fluid conductance through a tube is given by Poiseuille's formula:

$$
Q=\frac{\Delta P r^{4}}{8 L \nu}
$$

where $Q=$ fluid flow $\left(\mathrm{cm}^{3} \mathrm{sec}^{-1}\right)$

$$
\begin{aligned}
\Delta P & =\text { pressure drop }\left(\text { dynes } \mathrm{cm}^{-2}\right) \\
r & =\text { radius of tube }(\mathrm{cm}) \\
L & =\text { length of tube }(\mathrm{cm}) \\
\nu & =\text { fluid viscosity (poise) }
\end{aligned}
$$

It may be shown that, after rearranging the terms and assuming a viscosity of 0.007 poise, which is the viscosity of water, formula (2) may be expressed as:

$$
C=\frac{d^{4}}{l \times 3563}
$$

where $C=$ conductance $\left(\mu \mathrm{l} \mathrm{min}{ }^{-1} \mathrm{mmHg}^{-1}\right)$

$d=$ diameter of tube $(\mu \mathrm{m})$

$l=$ length of tube $(\mu \mathrm{m})$

Flow through an aperture is given by the equation (Happel and Brenner, 1965):

$$
Q=\frac{\Delta P r^{3}}{3 \nu}
$$

It is also possible to rearrange formula (4) and express it more conveniently as:

$$
C=\frac{d^{3}}{2099}
$$

According to Bill and Svedbergh the resistance, which is the inverse of the conductance, of each pore may be taken to be the resistance as calculated by formula (3) plus the resistance derived from formula (5). The values obtained for pore conductance and pore resistance by using this approach are shown in Table 3. Because of the small numbers involved, the pore counts in the 5 eyes in both the untreated group and the pilocarpine-treated group have been summed together to produce results for an equivalent area of $500000 \mu \mathrm{m}^{2}$ and extrapolated to $11 \mathrm{~mm}^{2}$ in each group.

The results are presented when all the pores are included and also when those pores above a diameter of $3 \mu \mathrm{m}$ have been omitted. Since the large pores have a considerable effect on the conductance, the inclusion of a potential artifact such as a largediameter pore will greatly increase the calculated conductance. Thus, while the pores of diameter 
Table 3 Calculated values for the conductance and resistance in the pore system in the lining endothelium of the trabecular wall of Schlemm's canal. The data were obtained from the grouped treated and untreated eyes and were calulated for an area of $500000 \mathrm{\mu m}^{2}$. The figures in parentheses are conversions to an area of $11 \mathrm{~mm}^{2}$

\begin{tabular}{|c|c|c|c|c|}
\hline & \multicolumn{2}{|c|}{$\begin{array}{l}\text { Pore conductance } \\
\left(\mu l \min ^{-1} \cdot \operatorname{mm} \mathrm{Hg}^{-1}\right)\end{array}$} & \multicolumn{2}{|c|}{$\begin{array}{l}\text { Pore resistance } \\
\left(\mathrm{mmHg} \min . \mu l^{-1}\right)\end{array}$} \\
\hline & All pores & Pores $<3 \mu m$ & All pores & Pores $<3 \mu m$ \\
\hline Treated & $\begin{array}{r}0 \cdot 985 \\
(21 \cdot 7)\end{array}$ & $\begin{array}{c}0 \cdot 837 \\
(18 \cdot 4)\end{array}$ & $\begin{array}{l}1.02 \\
(0.0462)\end{array}$ & $\begin{array}{l}1 \cdot 20 \\
(0 \cdot 0543)\end{array}$ \\
\hline Untreated & $\begin{array}{l}0 \cdot 104 \\
(2 \cdot 29)\end{array}$ & - & $\begin{array}{l}9 \cdot 61 \\
(0.437)\end{array}$ & - \\
\hline
\end{tabular}

greater than $3 \mu \mathrm{m}$ represent only $3 \%$ of the total number in the pilocarpine-treated group, they contribute $15 \%$ to the fluid conductance. The ratio between the pore resistance of the control group and the pore resistance of the pilocarpine-treated group is $\mathbf{9 . 4 7}$ when all the pores counted are included and 8.04 when only those less than $3 \mu \mathrm{m}$ are considered. Therefore according to this mathematical approach the effect of pilocarpine was to decrease the resistance in the pores by a probable factor of 9 .

In addition it can be seen from Table 3 that the resistance offered by the pore system in the untreated human eyes is $0.44 \mathrm{mmHg} \mathrm{min}^{-1} \mu \mathrm{l}^{-1}$. This value represents only one-sixth of the total trabecular resistance $\left(3 \mathrm{mmHg} \mathrm{min}^{-1} \mu \mathrm{l}^{-1}\right)$ as estimated by Grant (1958). These observations are in agreement with those of Bill and Svedbergh (1972), who considered that the bulk of the trabecular resistance is outside the canal endothelium and is probably in the underlying endothelial meshwork.

This work was supported by the Ross Foundation and the Scottish Hospital Endowments Research Trust (grant 442), and this help is gratefully acknowledget. We are indebted to the Anatomy and Engineering Departments of Glasgow University for the use of their scanning electron microscopes. It is also a pleasure to acknowledge the helpful co-operation of our clinical colleagues in the Scottish Western Region.

\section{References}

Bill, A. (1970). Scanning electron microscopic studies of the canal of Schlemm. Experimental Eye Research, 10, 214 218.

Bill, A., and Svedbergh, B. (1972). Scanning electron microscopic studies of the trabecular meshwork and the canal of Schlemm-an attempt to localise the main resistance to outflow of aqueous humor in man. Acta Ophthalmologica, 50, 295-320.

Grant, M. W. (1958). Further studies of facility of flow through the trabecular meshwork. Archives of Ophthalmology, 60, 523-533.

Grierson, I. (1976). The morphology of the outflow apparatus of the eye with particular reference to its structural appearance at various levels of intraocular pressure. Ph.D. Thesis. University of Glasgow.
Grierson, I., and Lee, W. R. (1974). Changes in the monkey outflow apparatus at graded levels of intraocular pressure: a qualitative analysis by light microscopy and scanning electron microscopy. Experimental Eye Research, 19, 21-33.

Grierson, I., and Lee, W. R. (1975). Pressure induced changes in the ultrastructure of the endothelium lining Schlemm's canal. American Journal of Ophthalmology, 80, 863-884.

Grierson, I., Lee, W. R., and Abraham, S. (1978). The effects of pilocarpine on the morphology of the human outflow apparatus. British Journal of Ophthalmology, 62, 302-313.

Happel, J., and Brenner, H. (1965). Low Reynolds Number Hydrodynamics. Prentice Hall: New Jersey.

Hoffman, F., and Dumitrescue, L. (1971). Schlemm's canal under the scanning electron microscope. Ophthalmic Research, 2, 37-45.

Holmberg, A., and Bárány, E. H. (1966). The effect of pilocarpine on the endothelium forming the inner wall of Schlemm's canal: an electron microscopic study in the monkey Cercopithecus aethiops. Investigative Ophthalmology, 5, 53-58.

Inomata, H., Bill, A., and Smelser, G. K. (1972). Aqueous humor pathways through the trabecular meshwork and into Schlemm's canal in the cynomolgus monkey (Macaca irus). An electron microscopic study. American Journal of Ophthalmology, 73, 760-789.

Kayes, J. (1967). Pore structure of the inner wall of Schlemm's canal. Investigative Ophthalmology, 6, 381-394.

Lee, W. R. (1971). The study of the passage of particles through the endothelium of the outflow apparatus of the monkey eye by scanning and transmission electron microscopy. Transactions of the Ophthalmological Societies of the United Kingdom, 91, 687-705.

Lee, W. R., and Grierson, I. (1974). Relationships between intraocular pressure and the morphology of the outflow apparatus. Transactions of the Ophthalmological Societies of the United Kingdom, 94, 430-449.

Lee, W. R., and Grierson, I. (1975). Pressure effects on the endothelium of the trabecular wall of Schlemm's canal: a study by scanning electron microscopy. Albrecht von Graefes Archiv für klinische und experimentelle Ophthalmologie, 196, 255-265.

McEwen, W. E. (1958). Application of Poiseuille's law to aqueous outflow. Archives of Ophthalmology, 60, 290-294.

Rohen, J. W., Lütjen, E., and Bárány, E. H. (1967). The relation between the ciliary muscle and the trabecular meshwork and its importance for the effect of miotics on aqueous outflow resistance. Albrecht von Graefes Archiv für klinische und experimentelle Ophthalmologie, 72, 23-47.

Segawa, K. (1973). Pore structures of the endothelial cells of the aqueous outflow pathway: scanning electron microscopy. Japanese Journal of Ophthalmology, 17, 133139.

Svedbergh, B. (1976). Aspects of the aqueous humor drainage. Functional ultrastructure of Schlemm's canal, the trabecular meshwork and the corneal endothelium at different intraocular pressures. Acta Universitatis Uppsaliensis Abstract of the Uppsala Dissertations in the Faculty of Medicine, 256, 1-71.

Tripathi, R. C. (1968). Ultrastructure of Schlemm's canal in relation to aqueous outflow. Experimental Eye Research, 7, 335-341.

Tripathi, R. C. (1971). Mechanisms of the aqeuous outflow across the trabecular wall of Schlemm's canal. Experimental Eye Research, 11, 116-121.

Tripathi, R. C. (1974). Comparative physiology and anatomy of the aqueous outflow pathway. In The Eye, Vol 5, pp. 163-356. Edited by H. Davson and L. J. Graham, Jr. Academic Press: New York and London. 